\title{
P. BOD
}

\section{Sur un modèle non-linéaire des rapports interindustriels}

Revue française d'automatique, d'informatique et de recherche opérationnelle. Recherche opérationnelle, tome 11, nº 4 (1977), p. 405-415.

<http://www.numdam.org/item?id=RO_1977_11_4_405_0>

(C) AFCET, 1977, tous droits réservés.

L'accès aux archives de la revue « Revue française d'automatique, d'informatique et de recherche opérationnelle. Recherche opérationnelle » implique l'accord avec les conditions générales d'utilisation (http://www.numdam.org/ legal.php). Toute utilisation commerciale ou impression systématique est constitutive d'une infraction pénale. Toute copie ou impression de ce fichier doit contenir la présente mention de copyright.

\section{Numdam}

Article numérisé dans le cadre du programme

Numérisation de documents anciens mathématiques

http://www.numdam.org/ 


\title{
SUR UN MODĖLE NON-LINÉAIRE DES RAPPORTS INTERINDUSTRIELS ( $\left.{ }^{*}\right)$
}

\author{
par P. Bod $\left({ }^{1}\right)$
}

\begin{abstract}
Résumé. - Une généralisation non-linéaire du modèle « input-output » ouvert et statique de W. Leontief est présentée.

Les hypothèses fondamentales du modèle sont identiques à celles adoptées par A. Nataf dans son article publié en 1960, avec l'exception que : au lieu de supposer un rendement croissant en général, des rendements quelconques sont admis.

Le noyau du modèle est une application : $F(x)$ de $R_{+}^{n}$ dans $R^{n}$ appartenant à la classe des $Z$-fonctions.

A l'aide d'un théorème de G. Wintgen, publié en 1964, on prouve les propriétés de minimalité et de complémentarité des $Z$-fonctions, observées pour la première fois par $A$. Tamir.

Les caractéristiques fondamentales du modèle sont les suivantes :

- il existe pour chaque demande finale accessible une production brute qui réalise cette demande à une dépense sociale minimum : $x_{0}$;

- si une condition de régularité est satisfaite à $x_{0}$, la fonction $F(x)$ possède dans ce point une jacobienne non-singulière avec une inverse non-négative;

- si on suppose le rendement croissant pour le système entier : la solution du modèle est unique.
\end{abstract}

Nous allons nous occuper d'une généralisation non-linéaire du modèle " intput-output» ouvert et statique de W. Leontief. Les hypothèses fondamentales que nous faisons, sont identiques à celles adoptées par André Nataf [3] dans son article publié en 1960 sous le titre : Systèmes économiques de production à rendement croîssant - avec une seule exception : notablement nous ne voulons pas supposer à priori un rendement croîssant en général; mais au lieu nous admettons des rendements quelconques.

Nous allons montrer qu'il existe dans le modèle pour chaque demande finale accessible une production brute qui réalise cette demande à une dépense sociale minimum.

Nous utiliserons dans notre analyse certains résultats pris de la théorie des programmes mathématiques "indifférents" ainsi que certaines notions et résultats de la théorie des ensembles ayant un élément plus petit.

Ce faisant - nous suivons deux objectifs particuliers. D'une part : nous voulons démontrer l'applicabilité des résultats mentionnés dans la généralisa-

(*) Reçu janvier 1976 .

(1) Institut de Mathématiques de l'Académie des Sciences de Hongrie.

R.A.I.R.O. Recherche opérationnelle/Operations Research, vol. 11, n 4, nov. 1977 
tion d'une famille importante des modèles macroéconomiques et d'autre part : nous indiquons que certains résultats publiés récemment sont plus ou moins des conséquences d'un vieux théorème publié par G. Wintgen en 1964 et qui semble être oublié [5]; [6].

Considérons une économie du type Leontief consistant en $n$ secteurs. Chaque secteur opère sur la base d'une technologie unique et produit une seule valeur d'usage homogène. Nous abstrayons de l'existence du commerce extérieure et essayons d'étudier à l'aide d'un modèle ouvert les rapports quantitatifs de la reproduction de l'économie globale.

A l'encontre de la supposition adoptée en générale dans les modèles inputoutput linéaires - selon laquelle les dépenses spécifiques sont constantes nous admettons que chaque secteur utilise la production de tous les secteurs pour sa propre activité et que les niveaux de ces dépenses sont déterminés par l'échelle de l'activité sectorielle.

Considérons maintenant le secteur $: j . n$ fonctions différentes caractérisent ses dépenses. Désignons par $f_{i j}\left(\xi_{j}\right) \cdot(i, j=1,2, \ldots, n)$ la quantité de produit venant du secteur : $i$ nécessaire pour la production d'une quantité $\xi_{j}$ dans le şecteur : $j$.

Nous avons ainsi $n^{2}$ fonctions de dépense tout ensemble dont nous faisons les hypothèses suivantes - raisonnables d'un point de vue économique :

i. les fonctions $f_{i j}\left(\xi_{j}\right)(i, j=1,2, \ldots, n)$ sont continuement dérivables;

ii. $f_{i j}(0)=0(i, j=1,2, \ldots, n)$

iii. $0 \leqq \xi_{j}^{1} \leqq \xi_{j}^{2}$ implique $f_{i j}\left(\xi_{j}^{1}\right) \leqq f_{i j}\left(\xi_{j}^{2}\right),(i=1,2, \ldots, n)$.

L'émission de chaque secteur sera utilisée selon la balance suivante :

$$
\xi_{i}=\sum_{j=1}^{n} f_{i j}\left(\xi_{j}\right)+\eta_{i}
$$

où $\eta_{i}$ dénote la partie de l'émission du secteur : $i$ désignée pour la demande finale.

Introduisons la notation suivante :

$$
f_{j}\left(\xi_{j}\right)=\left[\begin{array}{c}
f_{1 j}\left(\xi_{j}\right) \\
f_{2 j}\left(\xi_{j}\right) \\
f_{n j}\left(\xi_{j}\right)
\end{array}\right] \quad(j=1,2, \ldots, n)
$$

Ainsi nous pouvons écrire :

$$
x=\sum_{j=1}^{n} f_{j}\left(\xi_{J}\right)_{1}+y
$$

R.A.I.R.O. Recherche opérationnelle/Operations Research 
Soit :

$$
F(x)=x-\sum_{j=1}^{n} f_{j}\left(\xi_{j}\right)=\left[\begin{array}{l}
F^{1}(x) \\
F^{2}(x) \\
\vdots \\
F^{n}(x)
\end{array}\right]
$$

Nous gagnons l'équation fondamentale du modèle sous la forme :

$$
F(x):=y
$$

Il est évident que si les dépenses spécifiques sont constantes : - ça veut dire si

$$
f_{i j}\left(\xi_{j}\right):=a_{i j} \xi_{j}
$$

nous sommes chez le modèle linéaire de Leontief :

$$
F(x)=[E-A] x=y
$$

Pour éclairer la structure de la généralisation faite, introduisons quelques définitions :

DÉFInITION 1 : On dit - après W. C. Reinboldt, que l'application

$$
g(x): R_{+}^{n} \rightarrow R^{n}
$$

constituée des composants $g_{i}(x) ; \quad(i=1,2, \ldots, n)$ est extradiagonale antitonique si pour $\forall x \in R^{n}$ et pour $i \neq j(i, j=1,2, \ldots, n)$ les applications $\left.G_{i j}(\tau):=g_{i} x+\tau e_{j}\right)$ des applications de $R_{+}^{1} \rightarrow R^{1}$ sont des fonctions noncroîssantes de $\tau$.

DÉFINITION 2 : L'application $g(x)$ s'appelle diagonale isotonique, si pour $\forall x \in R_{+}^{n}$ les fonctions

$$
G_{i i}(\tau)=g_{i}\left(x+\tau e_{i}\right)
$$

sont non-décroissantes dans $\tau \geqq 0$

DÉFINITION 3 : On dit - après $\mathrm{A}$. Tamir - qu'une application

$$
g(x): R_{+}^{n} \rightarrow R^{n}
$$

est une $Z$-fonction si $g(x)$ est extradiagonale antitonique sur $R_{+}^{n}$. Une $Z$ fonction est une $M$-fonction si elle est « inverse-isotonique ». Cela veut dire :

$$
x ; y \in R_{+}^{n}: g(x) \leqq g(y) \Rightarrow x \leqq y
$$

Nous prouvons :

LeMme $1:$ L'application

$$
F(x)=\left[\begin{array}{l}
F^{1}(x) \\
F^{2}(x) \\
\vdots \\
F^{n}(x)
\end{array}\right]
$$


figurant dans l'équation fondamentale du modèle est une Z-fonction.

Preuve : Puisque

nous avons

$$
F^{i}(x),=\xi_{i}-\sum_{j=1}^{n} f_{i j}\left(\xi_{j}\right)
$$

$$
\begin{aligned}
F^{i}\left(x+\tau e_{k}\right) & =\xi_{i}-\sum_{\substack{j=1 \\
j \neq k}}^{n} f_{i j}\left(\xi_{j}\right)-f_{i k}\left(\xi_{k}+\tau\right) \\
& \leqq \xi_{i}-\sum_{\substack{j=1 \\
j \neq k}}^{n} f_{i j}\left(\xi_{j}\right)-f_{i k}\left(\xi_{k}\right)=F^{i}(x)
\end{aligned}
$$

Il est apparent que les $Z$-fonctions sont les généralisations de telles applications linéaires qui peuvent être caractérisées par des matrices $A=\left(a_{i j}\right)$ ayant $a_{i j} \leqq U$ s1 $i \neq j$.

Les $Z$-fonctions possèdent de nombreuses particularités, qui ont été montrées par Tamir dans sa note mentionnée. Tamir a étudié le problème de complémentarité relié à des $Z$-fonctions. [4]. Il a donné un algorithme pour la solution et pouvait montrer que si le problème possède une solution admissible - il possède aussi une solution qui est le plus petit élément dans l'ensemble des solutions admissibles et qui satisfait en même temps la condition de complémentarité.

Ces résultats de Tamir généralisent les résultats antérieurs de Cottle et Veinott $\mathrm{Jr}$. qui concernent le problème linéaire de complémentarité [1].

Nous allons montrer dans les suivants que ces propriétés indiquées sont conséquences directes d'un théorème de G. Wintgen publié en 1964 qui semble être oublié.

Le théorème de Wintgen se rapporte à l'idée de l'indifférence, introduite par lui-même.

Considérons une famille des problèmes d'optimisation dans laquelle il est donné l'ensemble des solutions admissibles : $L$ et une famille des fonctions objectives

$$
Z=\left\{z(x) \mid R^{n} \rightarrow R\right\}
$$

Le problème suivant de maximisation (minimisation) est un représentant de la famille de problèmes :

Il est à trouver le vecteur $\hat{x} \in R^{n}$ tel que

P.

$$
z(\hat{x})_{l}=\max _{x \in L}(\min ) z(x)
$$

où

$$
z(x) \in Z
$$

R.A.I.R.O. Recherche opérationnelle/Operations Research 
Définition 4 : Le problème $P$ est indifférent à l'égard de la famille des objectives $Z$ si $\exists x_{0} \in L$ tellement que

$$
z\left(x_{0}\right) \geqq(\leqq) z(x)
$$

pour $\forall x \in L$ et pour $\forall z(x) \in Z$.

Nous voulons définir $Z$ dans la forme suivante spécifique : soit donné un nombre fini des fonctions continues $z_{1}(x) ; z_{2}(x) ; \ldots z_{n}(x)$ atteignant toutes un maximum (minimum) fini sur $L$.

Soit

$$
Z^{\prime}=\left\{z(x) \mid z(x)=\sum_{i=1}^{k} \lambda_{i} z_{i}(x) ; \lambda_{i} \geqq 0\right\}
$$

La famille de fonction $Z^{\prime}$ se compose donc des combinaisons linéaires nonnégatives des fonctions données.

Les fonctions $z_{1}(x) ; z_{2}(x) ; \ldots ; z_{k}(x)$ réalisent une application de $R^{n}$ dans $R^{k}$. Dénotons l'image de l'ensemble $L$ dans $R^{k}$ transmis par cette application par $Z(L)$ et considérons le problème suivant :

Il est à trouver le vecteur $\hat{x} \in R^{n}$ tel que

$\mathrm{P}^{\prime}$.

$$
z(\hat{x})=\max _{x \in L}(\min ) z(x)
$$

où $z(x) \in Z$ !

Définition 5 : Soient $x$ et $y$ deux vecteurs dans $R^{n}$. Nous disons :

$$
\begin{array}{lll}
v=x \cup y & \text { si } & (v)_{i}=\max \left[\xi_{i} ; \eta_{i}\right]: \forall i \\
v=x \cap y & \text { si } & (v)_{i}=\min \left[\xi_{i} ; \eta_{i}\right]: \forall_{i}
\end{array}
$$

Nous prouvons maintenant le théorème de Wintgen :

Théorème 1 (Conditions suffisantes de l'indifférence): Le problème $P^{\prime}$ est indifférent à l'égard de la famille $Z^{\prime}$, si :

$$
x ; y \in L \Rightarrow Z(x) \cup Z(y) \in Z(L)
$$

respectivement :

$$
x ; y \in L \Rightarrow Z(x) \cap Z(y) \in Z(L)
$$

Preuve : Soient

$$
\begin{gathered}
z_{i}\left(\hat{x}_{i}\right)=\max _{x \in L}\left[z_{i}(x)\right] \\
(i=1,2, \ldots, k)
\end{gathered}
$$

$\hat{x}_{i} \in L(i=1,2, \ldots k)$ existent selon Définition 4. 
Formons le vecteur : $\bigcup_{i=1}^{k} Z\left(\hat{x}_{i}\right)$

$$
\bigcup_{i=1}^{k} Z\left(\hat{x}_{i}\right)\left[\begin{array}{c}
z_{1}\left(\hat{x}_{1}\right) \\
z_{2}\left(\hat{x}_{2}\right) \\
\dot{\cdot} \\
\dot{z_{k}}\left(\hat{x}_{n}\right)
\end{array}\right] \in Z(L)
$$

Il existe donc $x_{0} \in L$ tel que :

$$
Z\left(x_{0}\right)=\left[\begin{array}{ll}
\max & z_{1}(x) \\
\max & z_{2}(x) \\
\ldots & \cdots \\
\max & x_{n}(x)
\end{array}\right] ; x \in L
$$

Il est apparent que

$$
Z\left(x_{0}\right) \geqq Z(x): \forall x \in L
$$

Si $z(x) \in Z^{\prime}$ nous avons

$$
z\left(x_{0}\right)=\sum_{i=1}^{k} \lambda_{i} z_{i}\left(x_{0}\right) \geqq \sum_{i=1}^{k} \lambda_{i} z_{i}(x)=z(x) \quad \forall x \in L
$$

Par là nous avons montré l'existence d'un point $x_{n} \in L$ qui est une solution optimale sans regard à la spécifique fonction objective figurant dans $P^{\prime}$.

L'assertion du Théorème pour le cas du minimum peut être prouvée d'une manière analogue en appliquant l'opération $\cap$ au lieu de $\cup$.

DéfintTion 6 : On dit que $\hat{x}$ est l'élément plus grand (petit) dans $M \subset R^{n}$ si $\hat{x} \in M$ et $\hat{x} \geqq x: \forall x \in M(\hat{x} \leqq x: \forall x \in M)$.

Observons que $Z\left(x_{0}\right)$ est l'élément plus grand (petit) dans $Z(L)$ si $P^{\prime}$ est indifférent selon Définition 4.

DÉFINIT̀ion 7 : Donnée une application $f(x): R_{+}^{n} \rightarrow R^{n}$ et un vecteur $b \in R^{n}$ : le problème suivant est nommé " problème de complémentarité 》:

Il est à trouver un vecteur $x \in R^{n}$ tel que

i. $f(x)+b \geqq 0 ; x \geqq 0$

ii. $x^{\tau}[f(x)+b]=0$

Tous les vecteurs satisfaisant à $i$. sont des solutions admissibles; et une solution admissible est dite solution complémentaire si elle satisfait aussi à ii.

LEMME $\quad 2: \quad$ Soit $\quad L=\{x \mid f(x)+b \geqq 0 ; x \geqq 0\}$ $o \grave{u}$ $f(x): R_{+}^{n} \rightarrow R^{n} ; b \in R^{n}$. Supposons que $L \neq \varnothing ;|L|>1$. (L'ensemble $L$ possède au moins deux éléments distincts.) 
L'ensemble $L$ est fermé à l'opération $\cap$ si $f$ est une $Z$-fonction. C'est-à-dire : dans ce cas :

$$
x ; y \in L \Rightarrow x \cap y \in L
$$

Preuve :

$$
x ; y \in L \Rightarrow f_{i}(x)+\beta_{i} \geqq 0
$$

et

$$
f_{i}(y)+\beta_{i} \geqq 0 \quad(i=1,2, \ldots n)
$$

Soit $z=x \cap y$ comme $x \geqq 0 ; y \geqq 0 \Rightarrow z \geqq 0$. Nous montrons que $f_{i}(z)+\beta_{i} \geqq 0(i=1,2, \ldots n)$. Nous pouvons admettre sans que la généralité soit limitée que :

$$
(z)_{i}=\xi_{i}
$$

Soit définie une suite de point

où

$$
z=v_{0} ; v_{1} ; v_{2} ; \ldots ; v_{n}=x
$$

Nous prenons

$$
v_{k}=v_{k-1}+\tau_{k} e_{k}
$$

et

$$
\tau_{k}=0 \quad \text { si }(z)_{k}=\xi_{k}
$$

$$
\tau_{k}=\xi_{k}-\eta_{k} \quad \text { si }(z)_{k}=\eta_{k}
$$

Correspondant à notre définition : les fonctions $F_{i} k(\tau)$ sont non-croissantes et ainsi

$$
f_{i}\left(v_{k}\right)=f_{i}\left(v_{k-1}+\tau_{k} e_{k}\right) \leqq f_{i}\left(v_{k-1}\right)
$$

d'où la conclusion que $f_{i}(x) \leqq f_{i}(z)$ et

$$
f_{i}(x)+\beta_{i} \geqq 0 \Rightarrow f_{i}(z)+\beta_{i} \geqq 0
$$

Puisque cette relation est valable pour chaque $i$ nous avons reçu

$$
f(x)+b \geqq 0
$$

et ça signifie que $z \in L$

LeMme 3 : L possède l'élément plus petit si $f(x)$ est une $Z$-fonction continue et si $L \neq \varnothing$.

Preuve: Considérons la famille suivante de problèmes de programmation non-linéaire :

On cherche $\hat{x} \in R^{n}$ tel que $z(\hat{x})=\min z(x)$ :

$$
\begin{aligned}
x \in L & =\{x \mid f(x)+b \geqq 0 ; x \geqq 0\} \\
P^{\prime \prime} . \quad z(x) \in Z^{\prime \prime} & =\left\{\begin{array}{c}
z(x) \mid z(x)=\sum_{i=1}^{n} \lambda_{i} z_{i}(x) ; \lambda_{i} \geqq 0 \\
z_{i}(x)=\xi_{i}
\end{array}\right\}
\end{aligned}
$$

vol. $11, \mathrm{n}^{\circ} 4$, nov. 1977 
Preuve: $Z^{\prime \prime}$ est la famille des fonctions linéaires non-négatives. Les fonctions $z_{i}(x)$ réalisent maintenant l'application identique

$$
Z(x)=E x
$$

( $E$ est la matrice d'unité).

En vertu du Lemme 2 :

$$
x ; y \in L \Rightarrow Z(x) \cap Z(y)=x \cap y \in L=Z(L)
$$

L'ensemble $L$ est fermé car $f(x)$ est continue.

Si $L$ est compact : toutes les fonctions $z_{i}(x)$ prennent leur minimum sur $L$. Les conditions du Théorème 1 sont satisfaites, il existe donc $\hat{x} \in L$ où toutes les fonctions linéaires non-négatives ont leur minimum. Ce point est l'élément plus petit dans $L$.

Si $L$ n'est pas borné : nous choisissons un élément $\bar{x} \in L$ (qui existe bien sûr car $L \neq \varnothing)$. Considérons l'ensemble :

$$
L^{\prime}=\{x \mid x \in L ; x \leqq \bar{x}\} \subset L
$$

$L^{\prime}$ est évidemment compact, possède l'élément plus petit qui est à la fois le plus petit dans $L$.

LeMme $4:$ Si $x_{0}$ est l'élément plus petit dans $L$ et $f(x)$ est une Z-fonction continue : alors $x_{0}$ est une solution complémentaire.

Preuve: Supposons au contraire que la complémentarité n'est pas valide. Dans ce cas $\exists i$ tellement que

$$
\xi_{i}\left[f_{i}\left(x_{0}\right)+\beta_{i}\right]>0
$$

Si $\delta>0$ est assez petit - nous avons

$$
\bar{x}=x_{0}-\delta e_{i} \geqq 0
$$

Considérons les fonctions : $F_{i j}(\tau)$ partant du point $\bar{x} \in R_{+}^{n}$

$$
F_{j i}(\tau)=f_{j}\left[x_{0}-\dot{\delta} e_{i}+\tau e_{i}\right] \leqq f_{j}\left(x_{0}-\delta e_{i}\right)
$$

Prenant $\tau=\delta$

$$
F_{j i}(\delta)=f_{j}\left(x_{0}\right) \leqq f_{j}\left(x_{0}-\delta e_{i}\right)=f_{j}(\bar{x})
$$

Étant $f_{j}\left(x_{0}\right)+\beta_{j} \geqq 0$ : pour tous $j$ excepté $j=i$ nous avons

$$
f_{j}(\bar{x})+\beta_{j} \geqq 0
$$

Comme $f_{i}\left(x_{0}\right)+\beta_{i}>0$ et $f_{i}(x)$ est continue, $\delta$ peut être choisi si petit aussi que

$$
f_{i}(\bar{x})+\beta_{i}=f_{i}\left(x_{0}-\delta e_{i}\right)+\beta_{i} \geqq 0
$$

Donc $\bar{x} \in L$. Mais $\bar{\xi}_{i}<\xi_{i}^{0}$ et ça contredit à la súpposition que $x_{0}$ est l'élément le plus petit dans $L$ :

Après avoir discuté les caractéristiques des $Z$-fonctions nous retournons à notre modèle. 
Définition 8 : Une économie représentée par le modéle est dite « fonctionnelle » si $\exists \bar{x} \geqq 0$ tellement que

$$
F(\bar{x})>0
$$

DÉfINITION 9 : Nous appelons dans une économie fonctionnelle le vecteur $y_{0}>0$ une demande finale à atteindre si

$$
L_{y_{0}}=\left\{x \mid F(x) \geqq y_{0} ; x \geqq 0\right\} \neq \varnothing .
$$

Définition 10: La fonction numérique $\varphi(x)$ définie sur $R_{+}^{n}$ est dite une fonction de dépense sociale de la production si

i. $\varphi(0)=0$

ii. $\varphi(x)>0$ si $|x|>0$

iii. $\varphi(x)$ est isotonique, autrement dit :

$$
x^{\prime} \geqq x^{2} \Rightarrow \varphi\left(x^{\prime}\right) \geqq \varphi\left(x^{2}\right)
$$

THÉORÈME 2 : Il existe à toute demande finale à atteindre $: y_{0}$ une production brute : $x_{0}$ - tellement que

$$
F\left(x_{0}\right)=y_{0}
$$

et qui est optimal dans le sens que $x_{0}$ réalise la demande finale $y_{0}$ avec un minimum de dépense sociale de production.

Preuve : Si $y_{0}$ peut être atteint :

$$
L_{y_{0}}=\left\{x \mid F(x) \geqq y_{0} ; x \geqq 0\right\} \neq \varnothing .
$$

$F(x)$ étant une $Z$-fonction continue : $L y_{0}$ possède un élément plus petit : $x_{0}$ (Lemme 3) qui est une solution complémentaire (Lemme 4)

$$
x_{0}^{T}\left[F\left(x_{0}\right)-y_{0}\right]=0
$$

Nous montrons, que $x_{0}>0$. Supposons au contraire que $\xi_{i}=0$. Dans ce cas. la contrainte correspondant a la forme :

$$
\xi_{i}^{0}-\sum_{j=1}^{n} f_{i j}\left(\xi_{j}^{0}\right)-\eta_{i}^{0}<0
$$

c'est évidemment une contradiction, car dans cette forme $x_{0}$ ne peut pas être admissible.

La positivité de $x_{0}$ implique à cause de la complémentarité :

$$
F\left(x_{0}\right)-y_{0}=0 \quad \text { et } F\left(x_{0}\right)=y_{0}
$$

$x_{0}$ étant l'élément plus petit dans $L_{y_{0}}$ et puisque les fonctions de dépense sociale sont isotoniques : chaque $\varphi(x)$ atteint son minimum dans $x_{0}$.

THÉORÈME 3 : Si $x_{0}$ est l'élément plus petit dans $L_{y_{0}}$ et si une condition quelconque de régularité est satisfaite à $x_{0}$ : la fonction $F(x)$ possède une Jacobienne non-singulière dans ce point avec une inverse non-négative.

vol. $11, \mathrm{n}^{\circ} 4$, nov. 1977 
Preuve : $x_{0}$ étant l'élément plus petit dans $\mathrm{L}_{y_{0}} ; x_{0}$ est une solution optimale pour les problèmes suivants

$$
\begin{gathered}
x \in L_{y_{0}}=\left\{\begin{array}{c}
\min \xi_{i} \\
\left\{x \mid y_{0}-F\left(x_{0}\right) \leqq 0\right. \\
x \in R_{+}^{n}
\end{array}\right\} \\
(i=1,2, \ldots, n)
\end{gathered}
$$

Nous avons montré dans Théorème 2 que $x_{0}>0$ :c'est-à-dire $x_{0}$ est un point intérieur de $R_{+}^{n}$. Lorsque la régularité subsiste : les conditions nécessaires de l'optimalité sont acomplies. Il existe alors les vecteurs $u_{i} \geqq 0(i=1,2, \ldots, n)$ tel que $x_{0}$ et $u_{i}$ satisfont aux conditions Kuhn et Tucker : notamment à la condition selon laquelle le gradient de la fonction objective à $x_{0}$ est égal à une correspondante combinaison linéaire des gradients des contraintes actives. Mais à $x_{0}$ toutes les contraintes sont actives et nous avons

$$
\begin{gathered}
e_{i}=-\nabla\left[y_{0}-F\left(x_{0}\right)\right] u_{i}=\nabla F\left(x_{0}\right) u_{i} \\
(i=1,2, \ldots, n)
\end{gathered}
$$

Soit

$$
U=\left[u_{1} ; u_{2} ; \ldots ; u_{n}\right] \geqq 0
$$

Nous pouvons écrire :

et d'ici

$$
E=\nabla F\left(x_{0}\right) \cdot U
$$

$$
U=\left[\nabla F\left(x_{0}\right)\right]^{-1} \geqq 0
$$

ThÉOREMe 4 : Si on suppose ultérieurement le rendement croissant pour le système entier : la solution de l'équation

est unique.

$$
F(x)=y_{0}
$$

Preuve: L'hypothèse faite dit que les fonctions

$$
\frac{f_{i j}\left(\xi_{j}\right)}{\xi_{j}}(i, j=1,2, \ldots, n)
$$

sont non-croissantes; autrement dit : les fonctions de dépense $f_{i j}\left(\xi_{j}\right)$ sont concaves. Dans ce cas les sommes

$$
\sum_{j=1}^{n} f_{i j}\left(\xi_{j}\right)
$$

sont aussi concaves, tandis que les fonctions

$$
F^{i}(x)=\xi_{i}-\sum_{j=1}^{n} f_{i j}\left(\xi_{j}\right)
$$

sont convexes; alors tous les composants de $F(x)$ sont convexes.

R.A.I.R.O. Recherche opérationnelle/Operations Research 
Supposons à l'encontre du Théorème qu'il existe une seconde solution $\bar{x}$, différente de $x_{0}$.

La minimalité de $x_{0}$ implique $x_{0} \leqslant \bar{x} ; x_{0}$ et $\bar{x}$ satisfont à l'équation

$$
\left.\begin{array}{l}
F\left(x_{0}\right)=y_{0} \\
F(\bar{x})=y_{0}
\end{array}\right\} \Rightarrow F\left(x_{0}\right)-F(x)=0
$$

La convexité de $F(x)$ implique

$$
\nabla F\left(x_{0}\right)\left(\bar{x}-x_{0}\right) \leqq F(\bar{x})-F\left(x_{0}\right)=0
$$

L'inverse de Jacobienne est non-négative :

$$
\left[\nabla F\left(x_{0}\right)\right]^{1} \geqq 0
$$

Et nous recevons par multiplication :

$$
\bar{x}-x_{0} \leqq 0 \quad \text { et } \quad x_{0} \geqq \bar{x}
$$

ce qui contredit à $x_{0} \leqslant \bar{x}$

Remarque : L'unicité de la solution n'exige pas la convexité de l'application $F(x)$. Il suffit si $F(x)$ est localement quasi-convexe à $x_{0}$ concernant l'orthant non-négative.

\section{REFERENCES}

1. R. W. Cottle and A. F. Veinett Jr., Polyhedreal Sets having a Least Element, Math. Programming, 8, 1972, p. 238-249.

2. W. C. Reinboldt, On M Functions and their Applications in Nonlinear Gauss-Seidel Iterations and Network Flows, J. Math. Analysis Appl., 32, 1970, p. 274-307.

3. A. NaTAF, Systèmes économiques de production à rendement croissant, Publications de l'Institut de Statistique de l'Université de Paris, vol. IX, fasc. 2, 1960, p. 161-170.

4. A. TAMIR, Minimality and Complementarity Properties associated with Z-Functions and M-Functions, Math. Programming, 7, 1974, p. 17-31.

5. G. WINTGEN, Indifferente Optimierungsprobleme, M.K.O. Tagung, 1964, Konferenzprotokoll, Teil II, p. 3-6, Akademie Verlag, Berlin.

6. G. WINTGEN, Indiferente Optimierungsprobleme, in Operations Res. Verfahren 6, Verlag Anton Hain, 1969, p. 233-236. 